\title{
Towards radio transceiving in-vivo nano-robots
}

\author{
Shlomi Dolev ${ }^{1} \cdot$ Ramprasadh Narayanan $^{1}$ (i)
}

(c) Springer Nature Switzerland AG 2019

\begin{abstract}
An implementable model and simulation of an oscillating Carbon Nanotube (CNT) for radio communication in nanorobots is presented. A cylindrical CNT cantilever beam, operating under constant direct current supply, is forced to continuously oscillate by applying a controlled electromagnetic force. Oscillation parameters are computed and simulated, including the maximum tip displacement, the acting electromagnetic force and the dynamic response of the CNT, yielding a frequency of oscillation. We develop a model to predict the oscillation frequency of the cantilever beam based on its properties, including the device geometry. The primary functional component of the system is the electrically chargeable cantilever beam which oscillates due to its electric charge and discharge, where the discharge happens when the cantilever moves closer to the counter electrode. Thus, we provide an implementable design of in-vivo transceiver, suitable for future large-scale applications based on radio communicating nanorobots. A swarm of such nanorobots can be regarded as implementable programmable matter.
\end{abstract}

\section{Graphic abstract}

An implementable model and simulation of an oscillating Carbon Nanotube (CNT) for radio communication in nanorobots is presented. A cylindrical CNT cantilever beam, operating under constant direct current (DC) supply, is forced to continuously oscillate by applying a controlled electromagnetic force. Oscillation parameters are computed and simulated, including the maximum tip displacement, the acting electromagnetic force and the dynamic response of the CNT, yielding a frequency of oscillation. We develop a model to predict the oscillation frequency of the cantilever beam based on its properties, including the device geometry. The primary functional component of the system is the electrically chargeable cantilever beam which oscillates due to its electric charge and discharge, where the discharge happens when the cantilever moves closer to the counter electrode. Thus, we provide an implementable design of in-vivo transceiver, suitable for future large-scale applications based on radio communicating nanorobots. A swarm of such nanorobots can be regarded as implementable programmable matter.
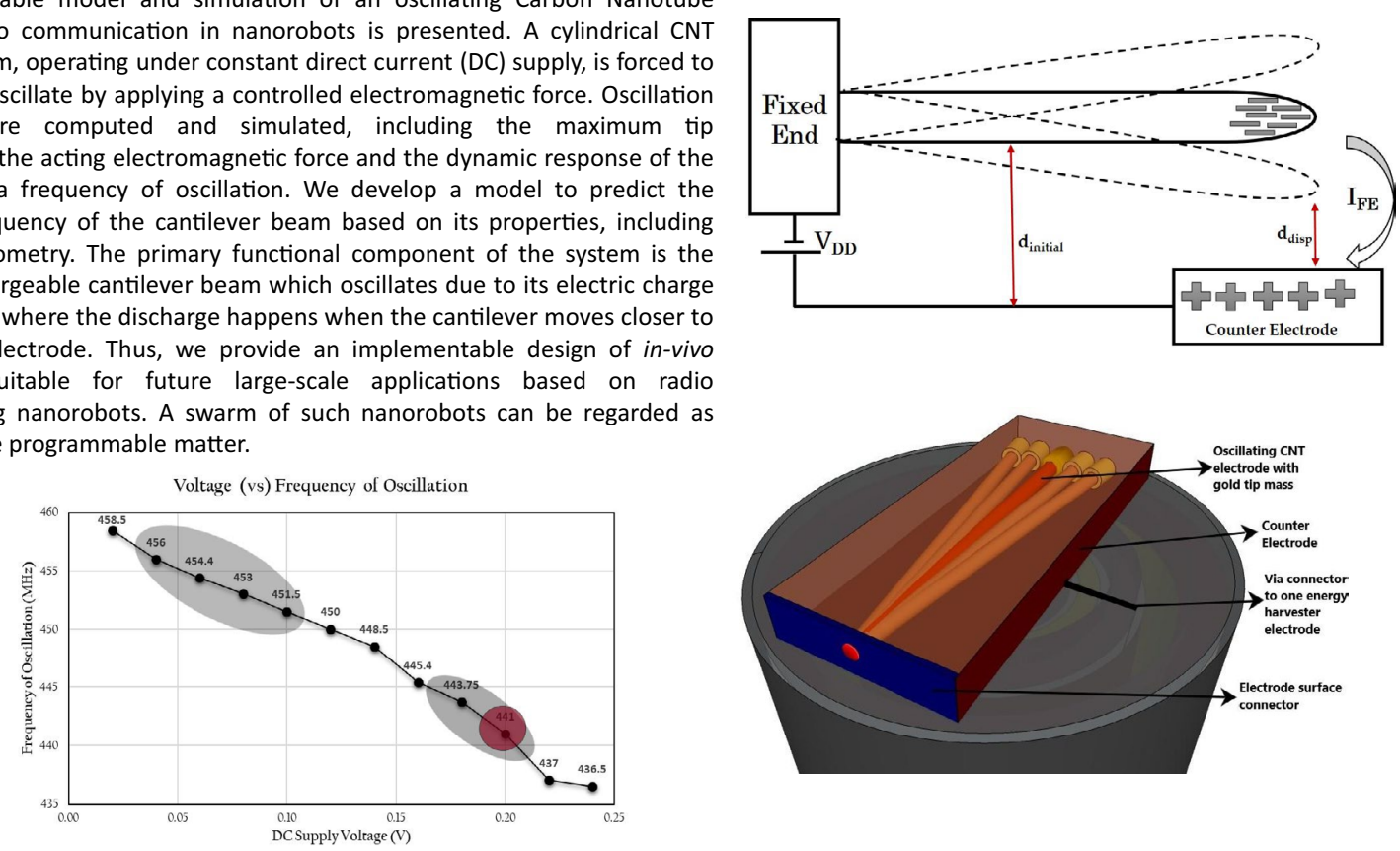

Ramprasadh Narayanan, narayan@cs.bgu.ac.il; Shlomi Dolev, dolev@cs.bgu.ac.il | 'Department of Computer Science, Ben-Gurion University of the Negev, Be'er Sheva, Israel.

SN Applied Sciences (2019) 1:969| https://doi.org/10.1007/s42452-019-1001-7 
Keywords Nano-Electro-Mechanical-Systems (NEMS) · Actuator · Cancer · Energy harvesting · Carbon Nanotube (CNT) detector $\cdot$ Tumor detection

\section{Introduction}

Nanorobotics is emerging as a growing research field with applications in medicine, surgery, intelligent coating, smart materials, to name a few. The promising advances in electronic fabrication and research on nanoparticles have attributed to vast improvements in this field. Until recently, the ability to realize theoretical concepts of nanorobotics into practice was limited to research stages. Recent research results in the field of DNA origami and computing [1-3], programmable matters $[4,5]$ and nanorobotic swarming systems [6] have provided evidences of implementable nanosystems. One prime example that can be defined as nanorobots would be the concept of programmable matters, defined as particles having certain computational capability that can either actively or passively involve in sensing, flocking and foraging. The ability of programmable matters to exhibit phenomenal changes to its properties upon action of chemical or bio-sensing $[7,8]$, or a combination of both [9], influences in realizing inorganic nanorobots for varied in-vivo sensing and actuation purposes.

In a previous work of ours, we provided a proof-of-concept design of an inorganic computational nanorobot. Figure 1 shows a representational image of a nanorobot with the intended oscillator design. The nanorobot is comprised of circular cylindrical bio-compatible electrodes to harvest and store charges. The charge mobility in the designed nanorobot is enabled through a bio-detection and actuation system, as described in our previous work [10]. The chamber structure shown in Fig. 1 is the design of the nano oscillator. The fixed end of the oscillator is connected to a thicker electrode wall, which in-turn is connected to the circular upper surface of one of the electrodes of the energy harvester. All but one of the side walls are coated with insulating medium to perfectly isolate the oscillator from fringe lumped elements. One side wall is connected to another terminal of the cylindrical energy harvester, thus completing the circuit (the oscillator acts as the load). The technical details of the oscillator design and the simulations conforming its working are explained in the sections below.

Radio communication using CNT cantilevers of similar geometry (in the $\mu m$ range) and conditions were demonstrated in $[11,12]$, where, resonant frequency of the device is proportional to the charge density and the geometry of the cantilever. In this work, we show an implementable model of radio communication in the nanorobots. We have provided the first implementable model of a down-scaled cantilever geometry which can be fabricated as a part of different nanodevices, but at the same time, under very low electrostatic power, that can be supplied by electrons harvested in blood $[7,10,13]$, an oscillation in the frequency range that fits in-vivo requirement is achieved.

\section{Methodology: modeling details}

Nano-Electro-Mechanical Systems (NEMS) based resonators [14] can be induced into oscillations which can sustain through continuous field emissions and applied Direct Current (DC) bias. NEM devices using CNTs have found their applications in sensing, high frequency oscillators and nanocircuits [15-17]. CNTs possess unique mechanical and electrical properties $[18,19]$ (including non-linear resonance [20]) which can be tweaked for futuristic new applications such as nano sensing devices in the bloodstream, biological sensing and detection [21, 22], elements for wireless devices such as nanoradio. They also show excellent capabilities for ballistic electron transport which can be exploited for fabricating short-length channels in electronic switches and increasing mass electron transport in biological sensing devices. These advantages of CNTs were exploited as cantilever beams for generating sustained mechanical oscillations to be used for radio transmission and reception [11, 12, 23, 24].

\subsection{Nano-radio transmitter}

We report a model and simulation of a field-emitting CNT cantilever oscillator which can be used as a transceiver in in-vivo medical devices. Using the model, we have elucidated the required geometry for achieving oscillation frequency, $f_{\text {osc }}$ in the MedRadio [25] bands, which defines the frequency spectrum for in-vivo medical devices. The MedRadio frequency spectrum is licensed for use in implantable medical devices like include implanted cardiac pacemakers, defibrillator and possibly, future nano transceivers. The frequency ranges allowed by the standard are: 401-406, 413-419, 426-432, 438-444, and 451-457 $\mathrm{MHz}$ ranges.

Figure 2 depicts the conceptual design for a CNT cantilever beam. A DC source is applied to the CNT of length $L$ and radius $r_{c}$, which initiates the field emission current, $I_{f e}$, between the electrodes. In our previous work [10], we have provided a model of an energy harvesting nanorobot structure. We detailed our estimation of the capacitance stored in the nanorobot by considering the glucose 


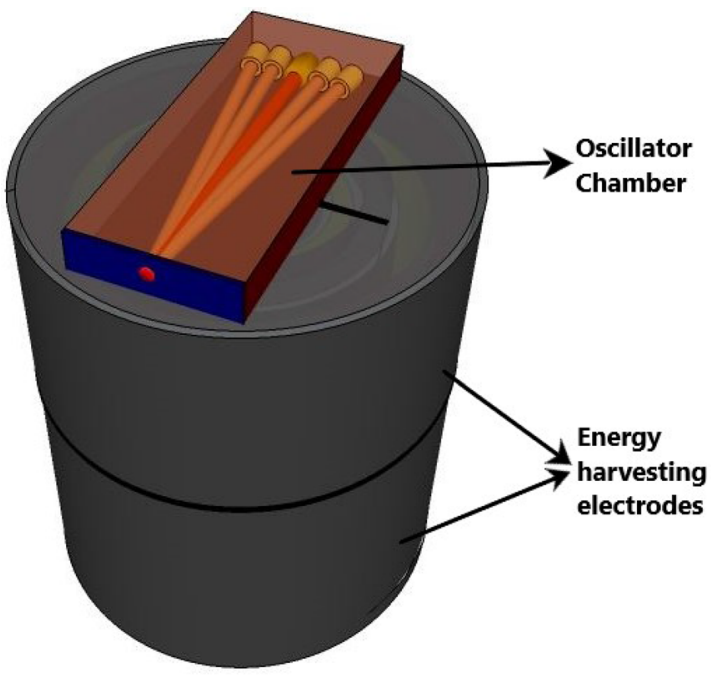

(a)

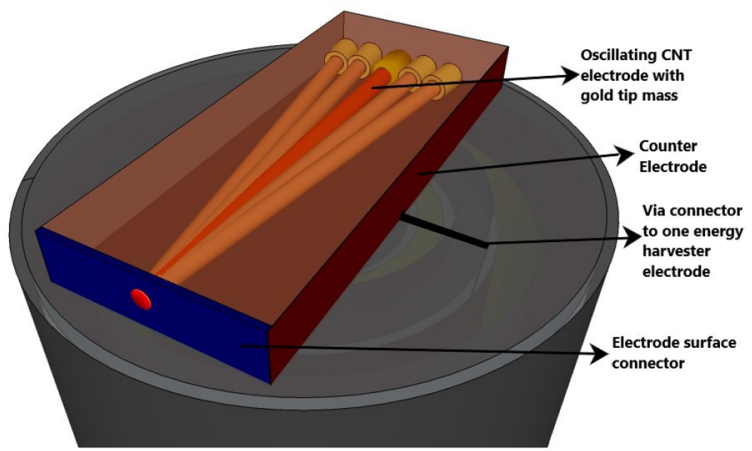

(b)

Fig. 1 a Total image of the nanorobot showing the energy harvester and the oscillator chamber, b Nano-oscillator chamber connected to the energy harvesting electrodes through the via lines (shown in black)

concentration of blood, velocity of blood flow, dimensional and material properties of the nanorobot. We estimated the stored capacitance to be $\approx 4.824 \times 10^{-16} \mathrm{~F}$. The provided energy harvesting capability can be used to power the movement of the CNT cantilever. The CNT is fixed to a non-movable electrode, separated from the counter electrode by an initial gap $d_{\text {initial }}$. This structure can be considered analogous to a parallel plate capacitor, wherein the capacitance of the plates is directly proportional to the electromagnetic force which bends the cantilever. The material and the structural geometry of the cantilever beam yields its structural stability, the tip displacement and the frequency of oscillation.

The cantilever is prescribed as a linear elastic material. A DC supply is swept at a constant interval. The electromagnetic force due to the capacitive voltage sweep resulted in the bending of the beam. Structural mechanics and electrostatics modules of COMSOL (License \#17075968) were used for computing the beam displacement and the acting electromagnetic force. Figure 3 depicts a COMSOL schematic structure of the beam.

The beam geometry is as follows. The length and radius of the CNT is set at $70 \mathrm{~nm}$ and $0.8 \mathrm{~nm}$, respectively. To accommodate the design for frequencies in the MedRadio frequency band [25], a gold mass is attached to the beam tip. Simulations without the extra gold mass resulted in frequency of oscillation much higher than the allowed MedRadio frequency for in-vivo devices. The dimensions of the tip mass and bare CNT were varied to fit the design in the needed frequency range. Once we add the mass, we could easily tune the frequency as needed, thus, when the device is implemented, frequency tuning can be done. Young's modulus of the CNT and the extra mass were set at $\approx 1000 \mathrm{GPa}$ [11] and $70 \mathrm{GPa}$, respectively. The length and radius of the cylindrical gold mass are both set at $1.7 \mathrm{~nm}$. Table 1 provides the geometry details used for the cantilever.

The design can be considered analogous to gold coated CNTs [28], wherein the tip of the CNT is coated with gold nanoparticles to increase the sensitivity of the nanoparticle, or in our case, the mass of the cantilever. The cantilever is enclosed in an air-filled chamber to increase the electrical insulation and to reduce the fringing effects between the capacitive elements. Prior simulations of a glucose energy harvesting nanodevice shows the maximum amount of potential generated to be $\approx 0.2 \mathrm{~V}$ [26]. This result was provided as an input potential between the two electrodes, the gold coated CNT and the counter electrode. The distance, $d_{\text {initial }}$ between the electrodes was fixed to $6.5 \mathrm{~nm}$. The multiphysics module in COMSOL handles the coupled calculations of structural mechanics and electrostatics.

On application of DC voltage, the nanotube tends to bend towards the counter electrode due to the accumulation of charges. The nanotube is oriented parallel to the surface of the counter electrode in order to achieve field emission due to constant switching of the CNT cantilever between the electromagnetic force, $F_{\text {elec }}$, and the elastic force $F_{\text {elastic }}$. This operation repeats due to continuous DC supply, forcing rapid charge-discharge cycle in the nanotube. It has to be noted that the cantilever will sustain stable oscillation as long as the electromagnetic 


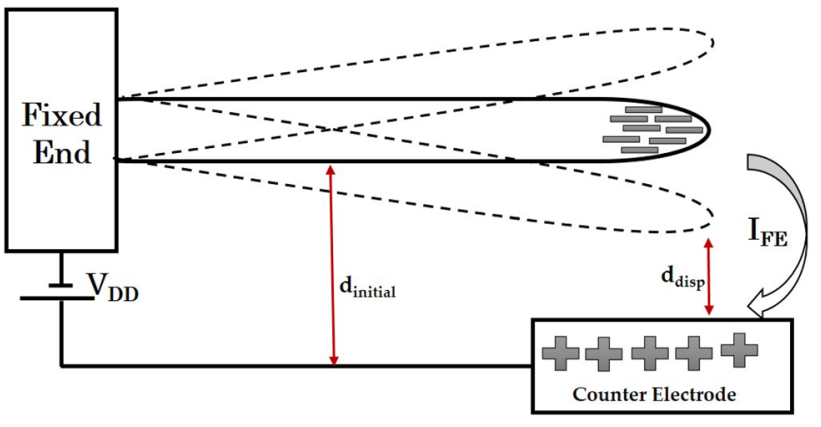

Fig. 2 Schematic of a uniform CNT cantilever oscillator

and the restoring elastic force are fixed. In the following paragraphs, we explain in detail our analysis and simulation results for the (i) Maximum tip displacement, $\delta_{m^{\prime}}$ (ii) Electromagnetic force, $F_{\text {elec }}$ and (iii) the Frequency of Oscillation, $f_{\text {osc }}$.

\section{Results}

We examined the maximum tip displacement, $\delta_{m}$ and the corresponding electromagnetic force, $F_{\text {elec }}$. With these results, we then calculated the frequency of oscillation, $f_{\text {osc }}$. Table 2 provides details of the force, surface displacement (both these values are obtained by COMSOL simulation), potential (input to the simulation) and the acceleration (numerically calculated) of the designed cantilever.

The total electromagnetic force can be approximated by the expression for the total electromagnetic force, $F_{\text {elec }}$ that is given in [11]. We calculated the total electromagnetic force and the maximum tip displacement acting on

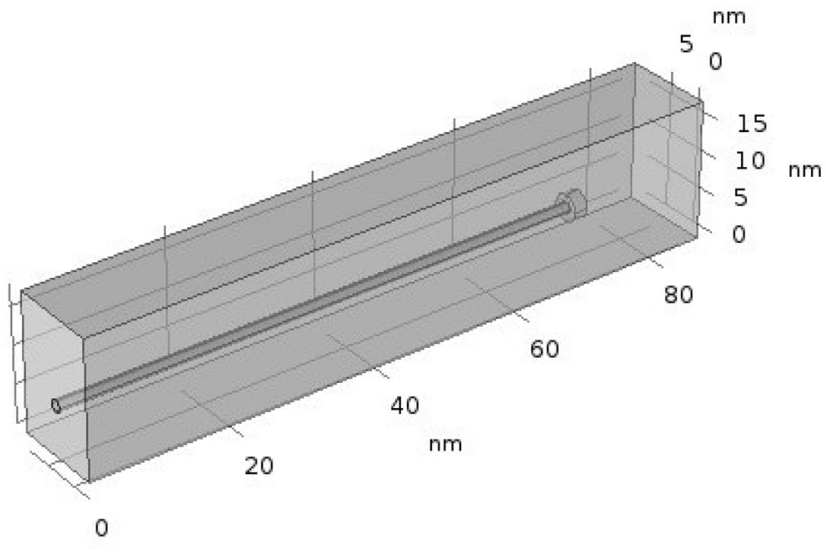

(a) the cantilever to be $\approx 2 \mathrm{pN}$ and $\delta_{m}=0.8 \mathrm{~nm}$, respectively. The simulated electromagnetic force and maximum tip displacement values are slightly different than the calculated values, wherein the difference in obtained values of $F_{\text {elec }}$ and $\delta_{m}$ can be attributed to the approximation made in the electromagnetic force acting on the side walls and tip of the cantilever beam. Figure $3 \mathrm{~b}$ shows the simulated structure and the obtained maximum displacement.

We then estimated the frequency of oscillation, $f_{\text {osc }}$ for the geometry and results provided in Table 2. Figure 4 shows the analysis of the frequency of oscillation as a function of applied DC voltage sweep. It is shown in the plots that the frequency of oscillation remains in the MedRadio band (shaded in gray) for the DC sweep range. We plotted the frequency of oscillation for varying length and radius of CNT cantilever, with a fixed geometry of the gold mass.

Table 1 Parameters used in numerical calculations and simulation

\begin{tabular}{ll}
\hline Parameters & Value \\
\hline Length of CNT Cantilever $(L)$ & $70-90 \mathrm{~nm}$ \\
Radius of CNT Cantilever $\left(r_{c}\right)$ & $0.8-2 \mathrm{~nm}$ \\
Length of gold mass & $1.7 \mathrm{~nm}$ \\
Radius of gold mass $\left(r_{g}\right)$ & $1.7 \mathrm{~nm}$ \\
Initial Gap between the electrodes $\left(d_{\text {initial }}\right)$ & $3-9 \mathrm{~nm}$ \\
Young's Modulus of the CNT $\left(E_{C N T}\right)$ & $1 \mathrm{TPa}[11]$ \\
Density of CNT cantilever $\left(\rho_{C N T}\right)$ & $2600 \mathrm{Kg} / \mathrm{m}^{3}[27]$ \\
Young's Modulus of the CNT $\left(E_{G o l d}\right)$ & $70 \mathrm{GPa}$ \\
Density of CNT cantilever $\left(\rho_{\text {Gold }}\right)$ & $19,300 \mathrm{Kg} / \mathrm{m}^{3}$ \\
$\epsilon_{0}$ & $8.854 \mathrm{pF} / \mathrm{m}$ \\
$V_{d c}$ & $0.2 \mathrm{~V}$ \\
\hline
\end{tabular}

Surface: Total displacement $(\mathrm{nm})$

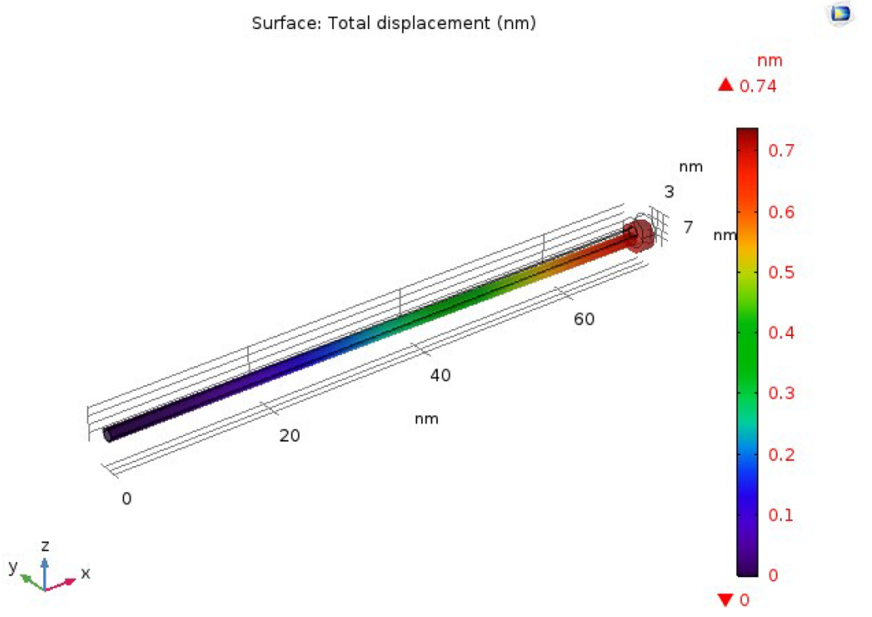

(b)

Fig. 3 a COMSOL design schematic. b Maximum tip displacement of $\approx 0.74 \mathrm{~nm}$ for a applied DC voltage of $0.2 \mathrm{~V}$, which can be harvested [26] 
Figure 5 shows the plot of frequency of oscillation as a function of the length and radius of CNT beam. For analyzing the frequency of oscillation, one parameter, either the length or the radius is kept as a constant, while varying the other. A legitimate combination for length, radius and frequency, $70 \mathrm{~nm}, 0.8 \mathrm{~nm}$ and $441 \mathrm{MHz}$ respectively, for a gold mass of both length and radius as $1.7 \mathrm{~nm}$, is shown in Fig. 5.

\section{Discussion}

\subsection{Analysis of $\boldsymbol{F}_{\text {elec }}$ and $\delta_{m}$}

The displacement of the beam and the electromagnetic force acting on it are a part of a feedback system, wherein the electromagnetic force initiates the displacement action. The decrease in distance between the electrodes, in-turn increases the electromagnetic force, thereby inducing higher displacement. This feedback loop system remains stable until the condition $F_{\text {elec }} \leq F_{\text {res }}$ is reached. Due to the cantilever-electrode parallel plate design, $F_{\text {elec }}$ is capacitive in nature.

Table 2 Simulated results of Electromagnetic force, $F_{\text {elec }}$ and Surface displacement, $\delta_{m}$. Length and radius of CNT: $70 \mathrm{~nm}$ and $0.8 \mathrm{~nm}$, respectively. Both the length and radius of gold mass are $1.7 \mathrm{~nm}$

\begin{tabular}{llll}
\hline $\begin{array}{l}\text { Electromagnetic } \\
\text { force }(\mathrm{N})\end{array}$ & Potential $(\mathrm{mV})$ & $\begin{array}{l}\text { Surface Disp. } \\
(\mathrm{nm})\end{array}$ & $\begin{array}{l}\text { Acceleration } \\
\left(\mathrm{m} / \mathrm{s}^{2}\right)\end{array}$ \\
\hline $2.3191 \mathrm{E}-12$ & 200.00 & 0.739 & $5.6824 \mathrm{E} 9$ \\
\hline
\end{tabular}

The maximum tip displacement, $\delta_{m^{\prime}}$ of a cantilever beam is given by [29],

$\delta_{m}=\frac{F_{\text {elec }}}{k_{s}}$

where, $k_{s}$ is the spring constant associated with the cantilever, given by $k_{s}=\frac{3 E I}{L^{3}}$, where, $l$ is the moment of Inertia given by $I=\frac{\pi r^{4}}{4}$. Therefore,

$\delta_{m}=\frac{F_{\text {elec }} L^{3}}{3 E I}=\frac{2.3191 \times 10^{-12} \times\left(70 \times 10^{-9}\right)^{3}}{3 \times 10^{12} \times \frac{\pi \times\left(0.8 \times 10^{-9}\right)^{4}}{4}}=0.8 \mathrm{~nm}$

During the movement of the cantilever away from base electrode, electrons are re-accumulated over the CNT and the electrostatic force is rebuilt, until the cantilever reaches the base electrode once more, completing a movement. Thus, the electrostatic force is used to avoid decay in the movement frequency of the cantilever. The spring behavior of the cantilever is analyzed incrementally to capture the non-linearity of its speed along each period of the periodical movement.

\subsection{Analysis of frequency of oscillation, $f_{\text {osc }}$}

The electromagnetic force can be given by the product of total mass $m$ and the acceleration $\ddot{\delta}_{t}$, as

$F=m \ddot{\delta}_{t}$

where $\ddot{\delta}_{t}$ is the acceleration of the nanotube with a displacement $\delta_{t}$, at time $t$. The total mass of the cantilever is calculated to be the sum of individual masses of single
Fig. 4 Frequency of oscillation for DC voltage sweep. The gray shaded areas show the authorized MedRadio frequency spectrum. The red shaded area shows the obtained frequency of oscillation for length and radius of CNT-70 $\mathrm{nm}$ and $0.8 \mathrm{~nm}$, respectively. Both the length and radius of the gold mass are $1.7 \mathrm{~nm}$

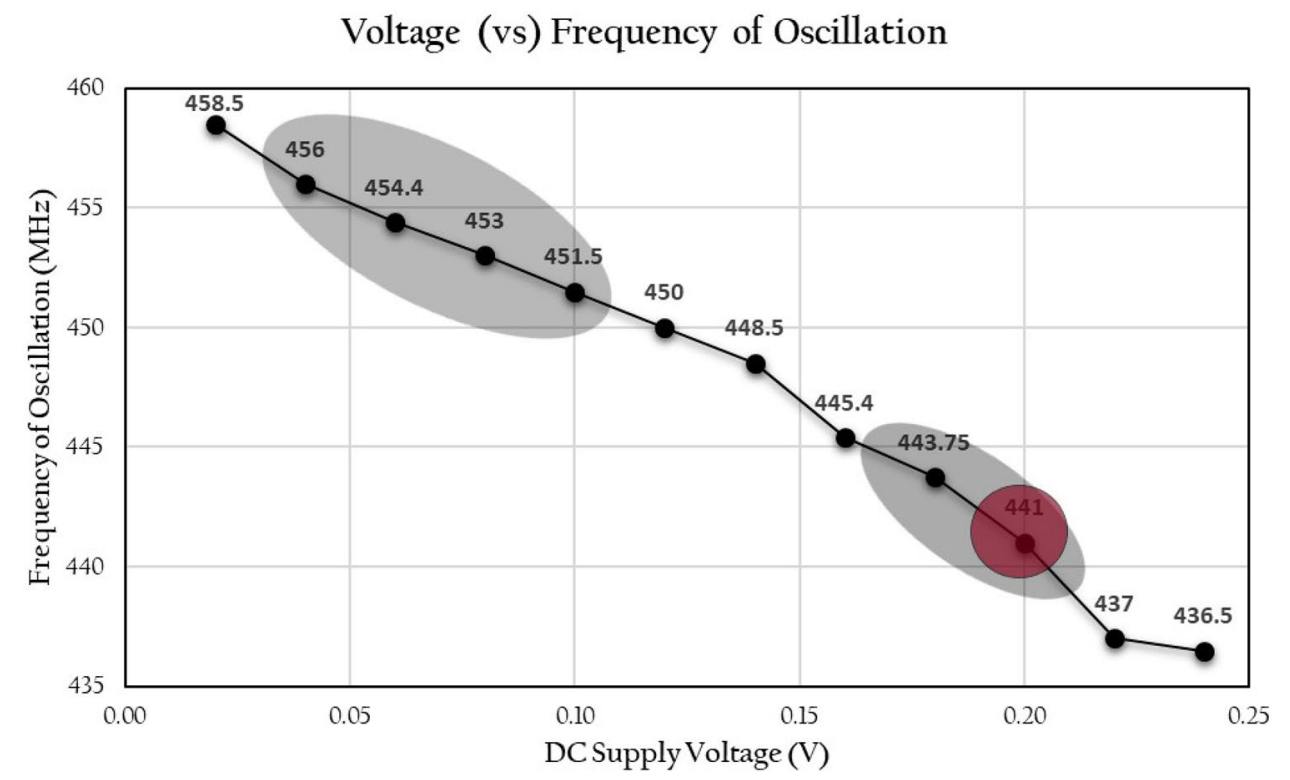

SN Applied Sciences 
Fig. 5 a $f_{\text {osc }}$ as a function of length of CNT ( $r=0.8 \mathrm{~nm}$, $\left.d_{\text {initial }}=6.5 \mathrm{~nm}\right) . \mathbf{b} f_{\text {osc }}$ as a function of radius of CNT $\left(L=70 \mathrm{~nm}, d_{\text {initial }}=6.5 \mathrm{~nm}\right)$
Length (vs) Frequency of Oscillation

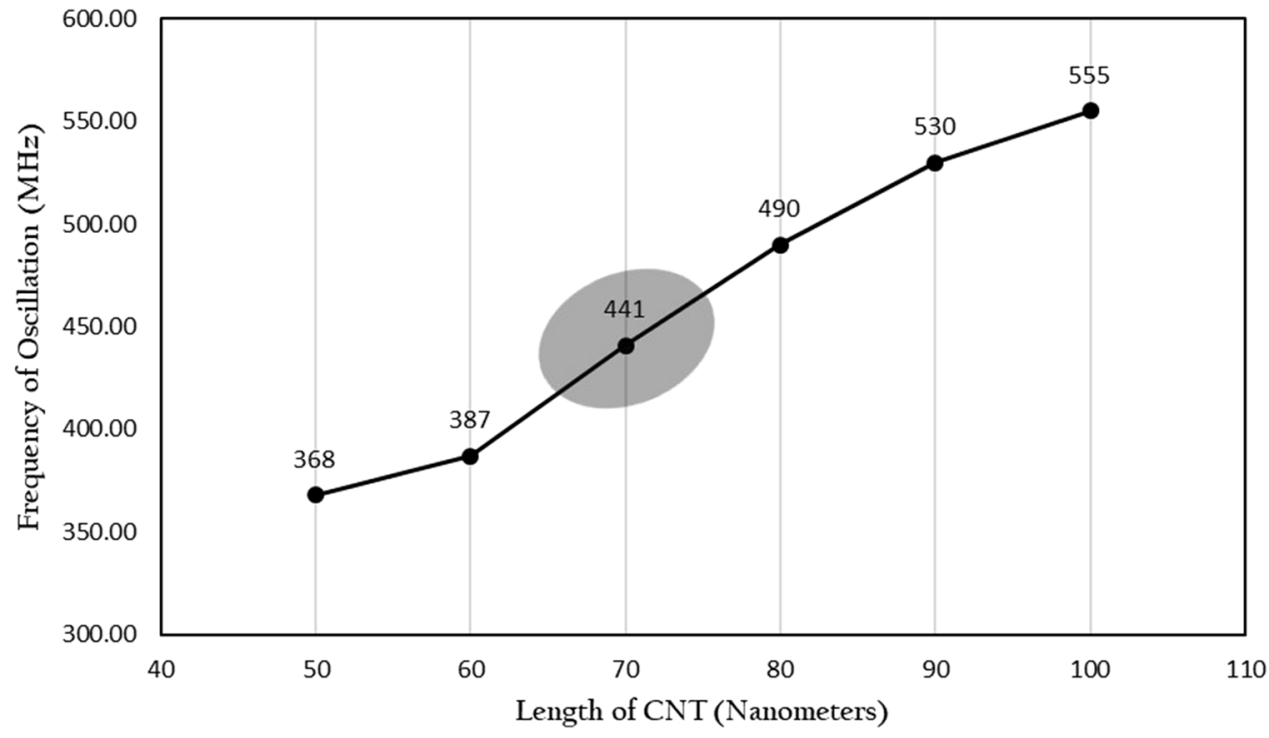

(a)

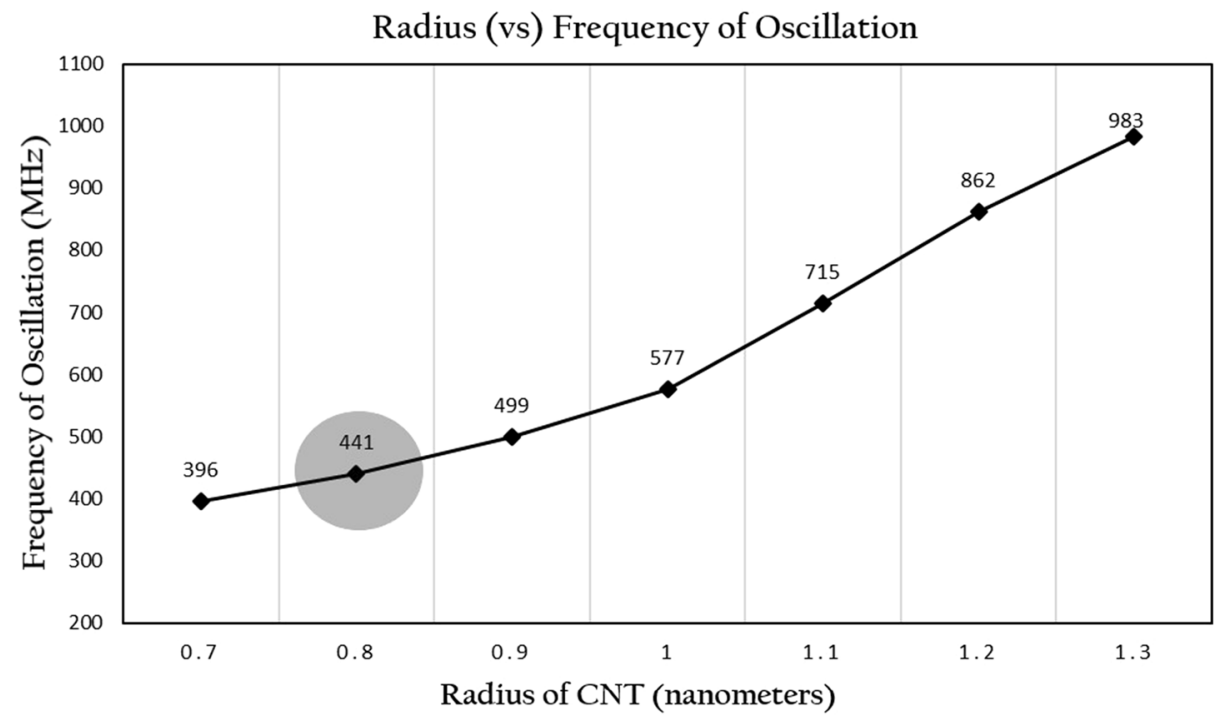

(b) walled nanotube and gold, which can be calculated from the density and volume of CNT and gold, respectively, as:

$$
\begin{aligned}
m_{\text {SWCNT }} & =\rho_{C N T} \times V_{C N T}=2600 \mathrm{~kg} / \mathrm{m}^{3} \times 32.78 \mathrm{~nm}^{3} \\
& =0.85 \times 10^{-22} \mathrm{Kg} \\
m_{\text {gold }} & =\rho_{\text {gold }} \times V_{\text {gold }}=19300 \mathrm{~kg} / \mathrm{m}^{3} \times 16.73 \mathrm{~nm}^{3} \\
& =3.22 \times 10^{-22} \mathrm{Kg}
\end{aligned}
$$

The maximum tip displacement $\delta_{m}$, can be expressed as a function of the acceleration and time as

$\delta_{t}=\delta_{0}+\dot{\delta}_{t} t+\frac{1}{2} \ddot{\delta_{t}} t^{2}$

\section{SN Applied Sciences}

where $\delta_{0}$ is the maximal displacement of the cantilever, where velocity is zero and the elastic force is maximal and $\dot{\delta}_{t}$ is the velocity of the cantilever at time $t$.

$\Longrightarrow \delta_{t}=\dot{\delta}_{t} t+\frac{1}{2} \ddot{\delta}_{t} t^{2}$

The subsequent velocities are given by

$\dot{\delta}_{(t+\Delta t)}=\dot{\delta}_{t}+\ddot{\delta}_{t} \Delta t$

The action of electromagnetic and elastic forces are constantly switched between each other, such that the condition of pull-in never occurs. 


$$
\begin{aligned}
& \text { at } t=0, \delta_{t}=0 \\
& \text { at } t_{1}=t+\Delta t, \delta_{t_{1}}=c_{1} \\
& \text { at } t_{2}=t 1+\Delta t, \delta_{t_{2}}=c_{2} \\
& \text { therefore, } t_{n}=t_{n-1}+\Delta t, \delta_{t_{n}}=\delta_{m}=c_{n} \\
& \Longrightarrow f_{\text {osc }}=\frac{1}{4\left(t_{1}+t_{2}+\cdots+t_{n}\right)}
\end{aligned}
$$

where, $\Delta t$ is the increase in time instance and $c_{1}, c_{2} \ldots c_{n}$ are the displacements of the cantilever for $t_{1}, t_{2} \ldots t_{n}$ respectively. We used growing number of iterations for estimating the differential equation, computing $t_{i}$ for each identical portion $c_{i}$, of the tip displacement. This resulted in significant stabilization of the result, obtaining $f_{\text {osc }}=441 \mathrm{MHz}$. The time it takes the cantilever to be parallel to the counter electrode with no elastic force, is multiplied by four, allowing the cantilever to resist to the velocity accumulated by the elastic force and then return to the parallel position, accumulating velocity and charges, that will further attract the cantilever to reach the maximal displacement once again. Therefore, the total distance oscillated by the cantilever is estimated to be $4 d_{\text {initial }}$. Thus, the time period of one oscillation (period for movement from maximal tip displacement position to the parallel position) is multiplied by 4 . This action of the cantilever is shown in Fig. 6.

As mentioned, the cantilever oscillating system can be considered as a feedback system wherein the electromagnetic force initiates (and maintains the amplitude for) the displacement action and the decrease in distance between the electrodes increases the electromagnetic force, thereby inducing higher displacement. Once the cantilever reaches the proximity of the counter electrode, it is discharged. At this point, the only significant force is the elastic force (we neglect the electromagnetic force acting on the beam on the way to reach point 3 in Fig. 6 , assuming there is a required CNT full charging time that is approximately the time it takes to reach point 3 . At point 3 , there is no action of velocity but only acceleration, due to the (beam string) elastic force acting on the cantilever.

The acceleration (which is decreased linearly, until it reaches zero when it is parallel to the counter electrodes) then yields the velocity (that is maximal at points 2 and 4 of Fig. 6). The velocity of the cantilever slows and accumulates in the elastic force of the cantilever, until it reaches point 3, where the velocity approaches zero. Then the acceleration due to the (opposite) tip dismantlement and elastic power of the beam yields a velocity towards points 4 , and this velocity together with the already charged CNT causes the cantilever to reach point 1. In fact, the amplitude of the oscillation does not decay due to the electrostatic extra power acting on the beam when reaching point 1 , once in every period. As the number of iterations is scaled up, the time calculated is closer to a continuous force change.

We have concentrated on including a radio device for our design, taking into account the possible energy harvesting and the frequency allowed for in-vivo transmission, tuning our design to respect the frequency limits specified by the MedRadio [25]. The addition of gold tip mass assist us in tuning the frequency of the CNT in order to obey the MedRadio band.

\section{Conclusion}

We have modeled and simulated a CNT based NEM cantilever beam with a geometry feasible to realize a fabricated device. We assume that repeated discharges of the cantilever due to close enough proximity of the cantilever to the other electrode, proximity that yield electron movement (discharge) from the cantilever to the other electrode. The proximity can be tuned by attaching a needle shaped structure from the gold tip mass, so that the inter-distance between the two electrodes is considerably reduced to a level for possible electron mobility. This theoretical model and simulation can be used as a proof-of-concept design towards fabrication. This work is part of the design of an inorganic computational nanorobot with a degree of autonomity in terms of bio-detection and actuation and communication. We envision future nanorobots with the capability of bio-detection, actuation and the ability to transmit status information to an outside entity. Millions of these tiny programmable matters can create vast amount of data to be used to further analyze and provide personalized treatment.

Furthermore, the oscillating source can be used as an input clock (the generated threshold of high and low signals) for realizing flip-flops and circuits embedded on the nanorobot to perform simple logical operations. As the

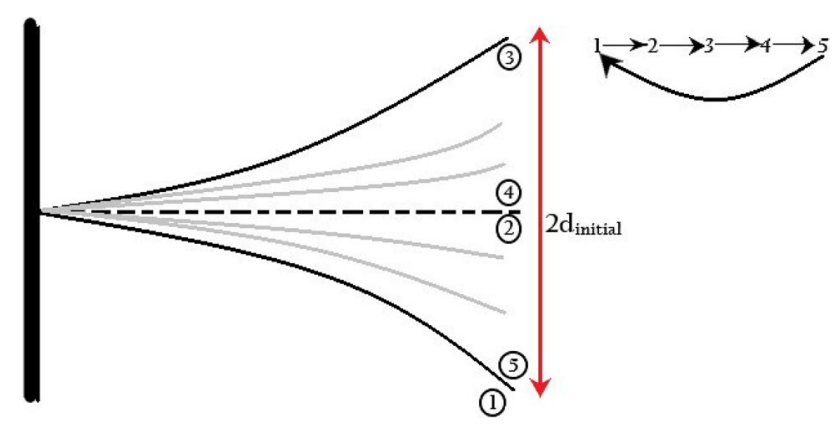

Fig. 6 Oscillation of Uniform CNT Cantilever 
cantilever can also be regarded as a part of a mechanical transceiver, it is possible that it will serve as a mean for output and input, to and from the nanorobots. We believe that our quantitative model and simulation establishes a comprehended design for plausible in-vivo medical radio devices.

Acknowledgements We thank the Lynne and William Frankel Center for Computer Science, the Rita Altura Trust Chair in Computer Science, the Kreitman School of Advanced Graduate Studies, BenGurion University of the Negev for their support. This research was also supported by a grant from the Ministry of Science \& Technology, Israel and German Research Funding Organization (DFG, Grant\#8767581199). The simulations were performed with COMSOL Multiphysics (License \#17075968). We also thank Prof. Zeev Zalevsky from Bar-Ilan University, Israel, Prof. Yonathan Sivan from Ben-Gurion University of the Negev and Prof. Tsu-Jae King Liu, University of California, Berkeley, for their useful comments and assistance. We also thank the anonymous reviewers for their useful comments.

\section{Compliance with ethical standards}

Conflict of interest The authors disclose no conflict of interest with another work or funding.

\section{References}

1. Thubagere AJ, Li W, Johnson RF, Chen Z, Doroudi S, Lee YL, Izatt G, Wittman S, Srinivas N, Woods D et al (2017) A cargo-sorting dna robot. Science 357(6356):eaan6558

2. Li S, Jiang Q, Liu S, Zhang Y, Tian Y, Song C, Wang J, Zou Y, Anderson GJ, Han J et al (2018) A dna nanorobot functions as a cancer therapeutic in response to a molecular trigger in vivo. Nat Biotechnol 36(3):258

3. Rothemund $P$ (2006) Folding dna to create nanoscale shapes and patterns. Nature 440(7082):297

4. Demaine E, Tachi T (2017) Origamizer: a practical algorithm for folding any polyhedron. In: LIPIcs-Leibniz international proceedings in informatics, vol 77. Schloss Dagstuhl-Leibniz-Zentrum fuer Informatik

5. Flocchini P, llcinkas D, Pelc A, Santoro N (2013) Computing without communicating: ring exploration by asynchronous oblivious robots. Algorithmica 65(3):562-583

6. Yu J, Wang B, Du X, Wang Q, Zhang L (2018) Ultra-extensible ribbon-like magnetic microswarm. Nat Commun 9(1):3260

7. Rapoport B, Kedzierski J, Sarpeshkar R (2012) A glucose fuel cell for implantable brain-machine interfaces. PLoS ONE 7(6):e38436

8. Liberti MV, Locasale J (2016) The Warburg effect: how does it benefit cancer cells? Trends Biochem Sci 41(3):211-218

9. Sarah nano-particle, 2016. http://www.innovex.co.il/_Uploads/ dbsAttachedFiles/NewPhasePresentationframeiNNOVEX201 6.pdf
10. Dolev S, Narayanan R, Rosenblit M (2019) Design of nanorobots for exposing cancer cells. Nanotechnology 30:315501

11. Weldon JA, Aleman B, Sussman A, Gannett W, Zettl A (2010) Sustained mechanical self-oscillations in carbon nanotubes. Nano Lett 10(5):1728-1733

12. Jensen K, Weldon J, Garcia H, Zettl A (2007) Nanotube radio. Nano Lett 7(11):3508-3511

13. Kerzenmacher S, Ducrée J, Zengerle R, Von Stetten F (2008) Energy harvesting by implantable abiotically catalyzed glucose fuel cells. J Power Sources 182(1):1-17

14. Craighead HG (2000) Nanoelectromechanical systems. Science 290(5496):1532-1535

15. Cimalla V, Niebelschütz F, Tonisch K, Foerster C et al (2007) Nanoelectromechanical devices for sensing applications. Sens Actuators B Chem 126(1):24-34

16. Chen C (2013) Graphene NanoElectroMechanical resonators and oscillators. Ph.D. thesis, Columbia University

17. Qian C, Peschot A, Osoba B, Ye ZA, Liu TK (2017) Sub-100 mv computing with electro-mechanical relays. IEEE Trans Electron Devices 64(3):1323-1329

18. Popov VN (2004) Carbon nanotubes: properties and application. Mater Sci Eng R Rep 43(3):61-102

19. Zhang A, Lieber CM (2015) Nano-bioelectronics. Chem Rev 116(1):215-257

20. Kim IK, Lee SI (2013) Theoretical investigation of nonlinear resonances in a carbon nanotube cantilever with a tip-mass under electrostatic excitation. J Appl Phys 114(10):104303

21. Poonia A, Dabas A, Chaudhary G, Kumar H, Singh KK (2014) Analysis of CNT based nano bio-sensor for virus detection. In: 14th IEEE international conference on nanotechnology, pp 747-751

22. Takayuki A, Shunichi S, Seiji A (2012) A multi-walled carbon nanotube cantilever for interaction force sensing in liquid. AIP Adv 2(1):012144

23. Weldon J, Jensen K, Zettl A (2008) Nanomechanical radio transmitter. Phys Status Solidi (b) 245(10):2323-2325

24. Rutherglen C, Burke $P$ (2007) Carbon nanotube radio. Nano Lett 7(11):3296-3299

25. Medical Device Radiocommunications Service (medradio) (2016). https://www.fcc.gov/medical-device-radiocommunicat ions-service-medradio

26. Dolev S, Rosenblit M, Narayanan RP (2018) Design of nanorobots for exposing cancer cells. In: 2018 IEEE 18th international conference on nanotechnology (IEEE-NANO), pp 1-5

27. Sugime $H$, Esconjauregui S, Yang J, D'Arsié L, Oliver RA, Bhardwaj S, Cepek C, Robertson J (2013) Low temperature growth of ultra-high mass density carbon nanotube forests on conductive supports. Appl Phys Lett 103(7):073116

28. Zhang RY, Olin H (2011) Gold-carbon nanotube nanocomposites: synthesis and applications. Int J Biomed Nanosci Nanotechnol 2(2):112-135

29. Cowper GR (1966) The shear coefficient in Timoshenko's beam theory. J Appl Mech 33(2):335-340

Publisher's Note Springer Nature remains neutral with regard to jurisdictional claims in published maps and institutional affiliations. 